\title{
Joint Stiffness Identification: a Three-Parameter Joint Model of Cantilever Beam
}

\author{
Sanjay B Ingole \\ Department of Mechanical Engineering, Government Polytechnic, Sadar, Nagpur (Maharashtra), INDIA-440 001
}

\begin{abstract}
Animesh Chatterjee
Department of Mechanical Engineering, Visvesvaraya National Institute of Technology, Nagpur (Maharashtra), INDIA-440 010
\end{abstract}

(Received 22 February 2014; accepted 26 August 2016)

In engineering applications, almost all structures are composed of substructures and parts that are joined together with a multitude of different connections (e.g., bolted, riveted, welded, etc.). It is known that the added flexibility introduced by the joint to a structure significantly affects its dynamic behavior. The need for accurate prediction of the dynamic characteristics of complex structures has led to extensive research on the identification of joint dynamic characteristics. In the present work, a structural joint have been modeled as a pair of translational and rotational springs and the frequency equation of the overall system has been developed using sub-structure synthesis. It is shown that by using the first few natural frequencies of the system, one can estimate the unknown stiffness parameters. The estimation procedure has been developed first for a two parameter joint model and then for a three parameter model in which cross coupling terms are also included. The validity of the proposed method is demonstrated numerically and experimentally.

\section{NOMENCLATURE}

$C \quad$ Composite system (sub-system I+II)

$F \quad$ Force corresponding to coordinate $X$

$K_{r} \quad$ Rotational stiffness of the joint

$K_{t} \quad$ Translational stiffness of the joint

$K_{t r} \quad$ Non dimensional cross coupling stiffness of the joint

$K_{x} \quad$ Non dimensional translational stiffness of the joint

$K_{y} \quad$ Non dimensional rotational stiffness of the joint

$K_{z} \quad$ Non dimensional cross coupled stiffness of the joint

$M \quad$ Moment corresponding to coordinate $\Theta$

S Sub-structure (sub system) I; joint system

pinv Generalised inverse of the matrix

$\mathrm{T} \quad$ Sub structure (sub-system) II, free-free beam

2-P Two parameter model of the joint system (model 1)

3-P Three parameter model of the joint system (model 2)

$q \quad$ Joint coordinate; translational

$\varphi \quad$ Joint coordinate; rotational

$\delta \quad$ Receptance function for the sub-system $\mathrm{S}$, joint system

$\eta \quad$ Receptance function for the sub-system $\mathrm{T}$, free-free beam

$\nu \quad$ Receptance function for the composite system C

$[\delta] \quad$ Frequency Response Function

(FRF) matrix for $\mathrm{S}$

$[\eta] \quad$ FRF matrix for $\mathrm{T}$

$[\nu] \quad$ FRF matrix for $\mathrm{C}$

$\{\hat{K}\} \quad$ Non dimensional joint parameter vector $\lambda \quad$ Non dimensional natural frequency of cantilever beam

$f_{1}--f_{6} \quad$ Functions defined in reference ${ }^{21}$

$\omega \quad$ Radian frequency

$f_{r} \quad$ Natural frequency $(\mathrm{Hz})$

\section{INTRODUCTION}

In the past, computational simulation of structure dynamics has become more accurate, reliable, and less expensive. However, the accuracy of dynamic simulation depends on the accuracy of the parameter of the structure. A real mechanical system usually consists of many components that are connected together through different joints. Modeling composite structures has become a challenging task due to uncertainty in system parameters, particularly those associated with structural joints. It is widely accepted that the behavior of the whole structure can be significantly affected by the way joints are modeled. In order to conduct an accurate dynamic analysis, it is first necessary to model the joints accurately and then identify their structural parameters. Basically, there are two different approaches to identify the joint properties; the model-based approach and the pure experimental approach.

The model-based approach is a hybrid approach where both experimental data and the theoretical model from the finite element (FE) method are used to identify the joint parameters. The objective of this approach is to minimize the error between the FE model and the experimental data with different techniques or algorithms. FE has become a standard method for structural analysis with a variety of FE software available for this purpose. However, some modeling error may be introduced in the theoretical models using the FE approximation. ${ }^{1-7}$ 
Although the pure experimental approach can use the experimental data in the time or frequency domain to identify the joint parameters, the frequency response functions are widely used in this approach. The main advantage of the experimental approach is that the theoretical modeling error of the structure can be avoided. However, the most troublesome problem of this approach is the unavoidable noise in the measurement data. The modal approach utilizes the modal parameters of a system, eigenvectors and eigenvalues to estimate the structural joint parameters. Although the modal approach is acknowledged as a useful tool for joint parameter identification, its usefulness has been hindered by the cost and difficulty involved in accurately estimating complete modal parameters for complicated structures. To alleviate this drawback in the modal approach, combined use of theoretical models together with modal parameters has often been attempted. Additionally, various model adjustment techniques to fit modal parameters have been proposed to identify structural parameters. ${ }^{8-12}$ These techniques, however, do not consider that a-priori information about the joint parameters is required. One of the first attempts to use the combination of experimental modal parameters with computer simulation to determine the dynamic response of a machine tool was made by Yoshimura. ${ }^{13}$ Pabst and Hagedorn ${ }^{14}$ demonstrated a system identification approach that is limited to the estimation of boundary condition by using measured modal data. Boundary condition of a cantilever beam was modeled by visco-elastic clamping, which is parameterized with translational and torsional stiffness with dampers parallel to each of the spring. They used two simple identification methods for determining the boundary parameters: first the characteristic equation was used for inverse analysis from measured eigen frequencies and in the second approach, the parameters were identified with the weighted least squares of modal residuals. Ahmadian et al. ${ }^{15}$ developed a new method to determine the boundary parameters based on the solution of reduced order characteristic equation using modal testing data. The effect of an elastic support was simulated by using a set of nodal forces on the boundary of a free structure. A relationship between nodal forces and nodal displacements was established at the boundary for each vibration mode. The support stiffness is also defined by a force-displacement relationship and the two systems of equations were then combined to identify the parameters of the boundary support. This method requires a FEM model before applying boundary conditions and natural frequencies of the physical structure constrained at its boundaries. Yang and Park proposed an efficient method for extracting joint structural parameters by combining the experimentally obtained incomplete FRFs with the sub-structure mathematical model. The unmeasured FRFs were estimated by solving an over determined set of linear equations derived from measured FRFs and the sub-structure mathematical model and then joint structural parameters were extracted by iterative output error algorithm. ${ }^{16}$

A joint identification method presented by Celic and Boltezar was based on the method developed by Ren and Beards. ${ }^{17-19}$ In the derivation they considered the effect of mass, stiffness, damping, and rotation. The FRF data from the accurately calibrated FE model were used for the estimation of the unmeasured FRFs and the identification of the damping in the joint. The system was ill conditioned at a higher frequency range but some useful results were obtained in the lower frequency range. A non-parametric model of the joint was used in the joint identification. Wang et al. presented a new joint properties identification method for improving the accuracy and robustness in solving the problem caused by the fact that only a part of the FRFs are experimentally measurable. To overcome the difficulty of partially measured FRFs, additional FRFs were added. In Wang et al., a new unmeasured FRFs estimation method is developed, which is capable of estimating all of the unmeasured FRFs using measured FRFs and modal calibrated sub-structure FEMs. Then both the estimated and measured FRFs are introduced into identification through a united form formula. Since all of the FRFs are included, the problems caused by measurement noise are significantly reduced and identification accuracy and robustness are improved but the dimension of the identification formula is larger than those in most of the previous methods. Thus this improvement is at the expense of computation cost. This is considered as the main limitation of the proposed method. ${ }^{20}$

In the present work, a simple and efficient method based on sub-structure synthesis for identification of boundary condition parameters is proposed. In most engineering structures, the friction joints are often tightly fastened so that their potential non linear behavior is suppressed and thus it is not significant. ${ }^{17}$ Hence, a linear model of the joint is considered in the present work. Only the first few measured natural frequencies are required in the identification procedure. This method is demonstrated for a cantilever beam with a two parameter (2-P) and three parameter (3-P) joint model, in which the boundary condition is initially modeled with a translational and rotational spring stiffness only and then additional cross coupled terms are added. The numerical simulation was carried out with non-dimensional stiffness parameters so that the results are applicable for any size or dimension of the actual test specimen. An experimental identification of joint stiffness for a real test structure is also presented the estimated joint parameters were then incorporated in the FE model. The FE model updated with the joint parameters gave natural frequencies in close agreement with the measured frequencies.

\section{THEORY OF SUB-STRUCTURE SYNTHESIS}

The sub-structure synthesis method is a model reduction technique whereby a relatively complex structure is analyzed as an assemblage of sub-structures. This method considers a complex structure as being constructed of interconnected substructures. The dynamics of the total structure is then synthesized by appropriately coupling the sub-structure dynamics. A sub-structure synthesis method translates sub-structure dynamics to total structure dynamics. The sub-structure dynamics can be individually studied and then appropriately coupled to form the complete structure.

The concept of sub-structure synthesis provides a formulation for the derivation of Frequency Response Functions (FRFs) of a composite system from the knowledge of sub- 


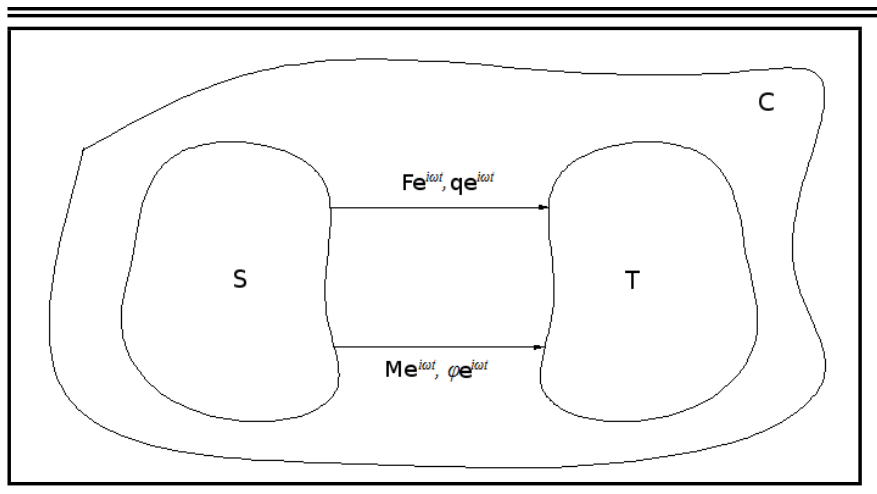

Figure 1. A composite system $\mathrm{C}$ with sub-systems $\mathrm{S}$ and $\mathrm{T}$.

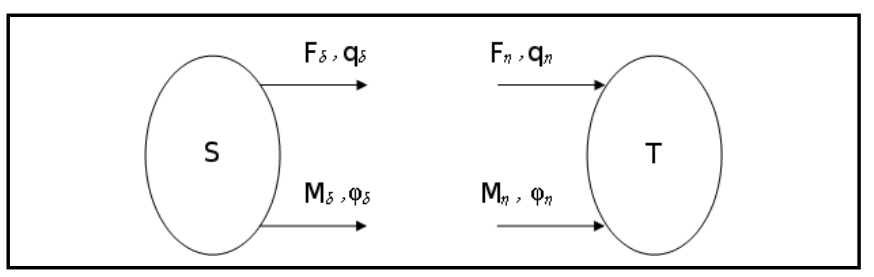

Figure 2. Force and displacements acting on the sub-systems S and T.

system FRFs. A composite system $\mathrm{C}$ shown in Fig. 1 consists of two sub-systems $\mathrm{S}$ and $\mathrm{T}$ connected by two joint coordinates $q$ and $\varphi$ at the interface. The force and moment at the interface of two sub-systems are $\mathrm{F}$ and $\mathrm{M}$.

Figure 2 shows the sub-systems separated with corresponding displacements and forcing functions at the interface.

The dynamic equilibrium of sub-systems $\mathrm{S}$ and T separately, the compatibility requirement of forces, and the displacements at the joint co-ordinate, gives the frequency equation for the composite system in terms of receptances of individual subsystems as,

$$
\left(\delta_{11}+\eta_{11}\right)\left(\delta_{22}+\eta_{22}\right)-\left(\delta_{12}+\eta_{12}\right)^{2}=0 ;
$$

where, $\delta_{11}, \delta_{22}$ and $\eta_{11}, \eta_{22}$ are the direct receptance functions and $\delta_{12}, \delta_{21}$ and $\eta_{12}, \eta_{21}$ are the cross receptance functions of sub-systems $\mathrm{S}$ and $\mathrm{T}$, respectively. The derivation of frequency Eq. (1) can be obtained from Ingole and Chatterjee. ${ }^{21}$

Thus, if the FRFs at the interface co-ordinates are known for the individual sub-systems, then the natural frequencies of the composite system can be obtained by solving the frequency Eq. (1). If sub-system $\mathrm{T}$ is a structural component and subsystem $\mathrm{S}$ is a joint characterized by the joint parameters $\delta_{i j}$, then Eq. (1) provides a basis to study the effect of joint parameters on the system natural frequencies. However, in the present work, Eq. (1) was exploited for an inverse analysis, where one can estimate the joint parameters from the over determined set of measured frequency data, using multi-linear regression technique. ${ }^{22}$

The structure under demonstration in this paper consists of a beam with uniform cross section, clamped at one end with a bolt. Two models of the joint are considered, so called, 2parameter and 3-parameter model.

Model 1: $\mathbf{S}_{k 1}=\left[\begin{array}{cc}K_{t} & 0 \\ 0 & K_{r}\end{array}\right]$, 2-parameter model.

Model 2: $\mathbf{S}_{k 2}=\left[\begin{array}{cc}K_{t} & K_{t r} \\ K_{r t} & K_{r}\end{array}\right]$, 3-parameter model.

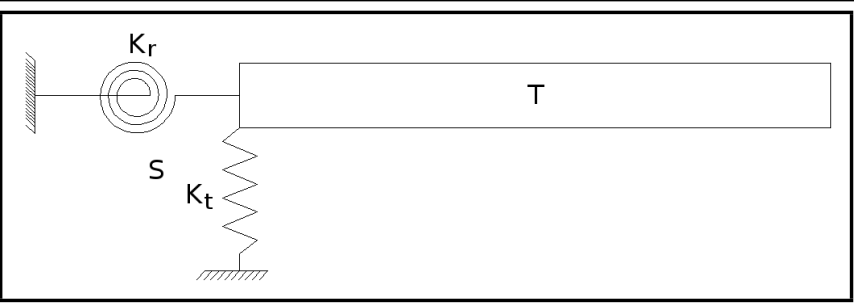

Figure 3. A cantilever beam modeled with elastic support at the fixed end.

\section{A THEORY FOR A TWO PARAMETER JOINT MODEL OF A CANTILEVER BEAM}

A boundary condition of a cantilever beam is modeled with elastic constraint represented by a translational spring (stiffness $K_{t}$ ) and a rotational spring (stiffness $K_{r}$ ) as shown in Fig. 3, thus designated as a 2-parameter joint model.

A cantilever beam is considered as a composite system consisting of elastic springs as sub-system $\mathrm{S}$ and a free-free beam as sub-system T. A method for derivation of frequency equation for 2-parameter joint model of the cantilever beam is presented in detail by Ingole and Chatterjee. ${ }^{21}$ However it is required to demonstrate the method here to establish need of 3parameter model discussed in section 4 . The frequency equation for 2-parameter joint model can be obtained as ${ }^{21}$

$$
K_{x} \cdot K_{y}+K_{x} \lambda \frac{f_{5}}{f_{4}}-K_{y} \cdot \lambda^{3} \cdot \frac{f_{6}}{f_{4}}-\lambda^{4} \frac{f_{3}}{f_{4}}=0
$$

where $f_{4}=1+\cos (\lambda) \cosh (\lambda) ; K_{x}=\frac{K_{t}}{\left(E I / L^{3}\right)}$ and $K_{y}=$ $\frac{K_{r}}{(E I / L)}$. The functions $f_{3}, f_{5}$ and $f_{6}$ are same as defined by Ingole and Chatterjee. ${ }^{21}$

If we experimentally measure 'n' natural frequencies $\omega_{i}(i=$ $1,2 \ldots n)$, and $\lambda_{j}, \lambda_{k}$ be the values corresponding to $\omega_{j}$ and $\omega_{k}(j, k=1,2 \ldots n ; j \neq k)$, then Eq. (2) gives two equations corresponding to $\lambda=\lambda_{j}, \lambda_{k}$, subtracting these two equations gives, an equation which can be represented as,

$$
\mathbf{T}_{i} \cdot K_{x}+\mathbf{U}_{i} K_{y}=\mathbf{V}_{i}
$$

Equation (3) is a linear equation in two unknowns $K_{x}$ and $K_{y}$; $\mathbf{T}_{i}, \mathbf{U}_{i}$ and $\mathbf{V}_{i}$ are the coefficients which can be computed from the pair $\lambda_{j}, \lambda_{k}$. For each pair of $\lambda=\lambda_{j}, \lambda_{k}$, we get one equation from Eq. (3). Thus if we measure $\mathrm{n}$ natural frequencies, we get ${ }^{n} C_{2}$ number of equations in two unknowns $K_{x}$ and $K_{y}$. Since number of equations will be generally more than the number of unknowns, which are two here, one can employ the method of multi linear regression to get a least square error estimate of the unknown joint stiffness parameters. ${ }^{22}$

Taking $r={ }^{n} C_{2}$, Eq. (3) can be written as,

$$
\begin{array}{r}
{\left[\begin{array}{lr}
\mathbf{T}_{1} & \mathbf{U}_{1} \\
\mathbf{T}_{2} & \mathbf{U}_{2} \\
-- & -- \\
\mathbf{T}_{r} & \mathbf{U}_{r}
\end{array}\right]\left\{\begin{array}{l}
K_{x} \\
K_{y}
\end{array}\right\}=\left[\begin{array}{c}
\mathbf{V}_{1} \\
\mathbf{V}_{2} \\
-- \\
\mathbf{V}_{r}
\end{array}\right]} \\
\text { or }[\mathbf{T U}]\{\hat{\mathbf{K}}\}=[\mathbf{V}] .
\end{array}
$$

The least square error criterion gives the best estimate of the joint parameter vector as,

$$
\{\hat{\mathbf{K}}\}=\operatorname{pinv}(\mathbf{T U}) \mathbf{V}
$$

where pinv is the generalized inverse of a matrix. ${ }^{23}$ 


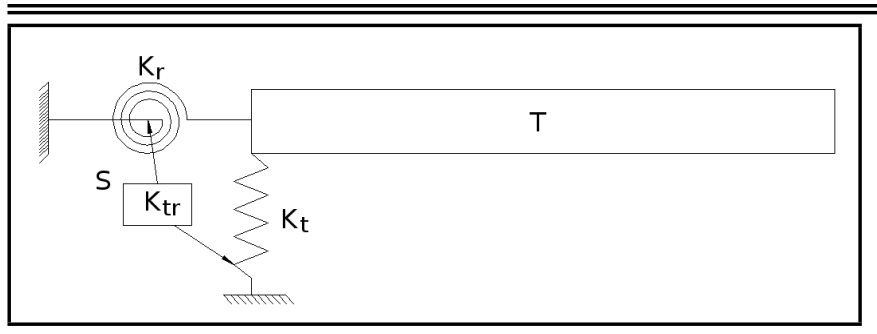

Figure 4. A cantilever beam modeled with elastic support at the fixed end (3-P).

\section{A THREE PARAMETER JOINT MODEL OF A CANTILEVER BEAM: THEORY}

A cantilever beam with boundary condition (fixed end) modeled as an elastic support consisting of a translational, rotational and cross coupled terms, thus designated as 3-parameter (3-P) model is shown in Fig. 4. The concept of sub-structure synthesis, discussed in section 2 , is used to derive the frequency equation of the composite system consisting of a freefree beam interfaced with a joint at one end.

A 3-P model of joint is represented by a $(2 \times 2)$ matrix which include cross coupled terms. The FRF matrix for joint system $\mathrm{S}$ is then represented by $\delta=\left[\begin{array}{cc}1 / K_{t} & 1 / K_{t r} \\ 1 / K_{r t} & 1 / K_{r}\end{array}\right]$ The FRF matrix $\eta$ for $\mathrm{T}$ is same as discussed in section 3 .

Now, simplifying frequency equation by substituting direct and cross receptances of sub-structure $\mathrm{S}$ and $\mathrm{T}$ in Eq. (1), and further defining the joint stiffness parameters as nondimensional parameters $K_{x}, K_{y}$ and $K_{z}$ gives,

$$
\begin{array}{r}
\frac{1}{K_{x}}\left\{\frac{1}{\lambda}\left(\frac{f_{6}}{f_{3}}\right)\right\}-\frac{1}{K_{y}}\left\{\frac{1}{\lambda^{3}}\left(\frac{f_{5}}{f_{3}}\right)\right\}- \\
-\frac{1}{K_{z}}\left\{\frac{2}{\lambda^{2}}\left(\frac{f_{1}}{f_{3}}\right)\right\}= \\
=\left\{\frac{1}{\lambda^{4}}\left(\frac{f_{4}}{f_{3}}\right)\right\}-\left(\frac{1}{K_{x} K_{y}}-\frac{1}{K_{z}^{2}}\right) ;
\end{array}
$$

where, $K_{z}=\frac{K_{t r}}{\left(E I / L^{2}\right)}=\frac{K_{r t}}{\left(E I / L^{2}\right)}$.

For each pair of measured natural frequency Eq. (6) gives two equations corresponding to $\lambda=\lambda_{j}, \lambda_{k}(j \neq k)$, subtracting these two equations gives,

$$
\begin{gathered}
\frac{1}{K_{x}}\left\{\frac{1}{\lambda_{j}}\left(\frac{f_{6}}{f_{3}}\right)_{\lambda=\lambda_{j}}-\frac{1}{\lambda_{k}}\left(\frac{f_{6}}{f_{3}}\right)_{\lambda=\lambda_{k}}\right\}+ \\
+\frac{1}{K_{y}}\left\{\frac{1}{\lambda_{k}^{3}}\left(\frac{f_{5}}{f_{3}}\right)_{\lambda=\lambda_{k}}-\frac{1}{\lambda_{j}^{3}}\left(\frac{f_{5}}{f_{3}}\right)_{\lambda=\lambda_{j}}\right\}+ \\
+\frac{1}{K_{z}}\left\{\frac{2}{\lambda_{k}^{2}}\left(\frac{f_{1}}{f_{3}}\right)_{\lambda=\lambda_{k}}-\frac{2}{\lambda_{j}^{2}}\left(\frac{f_{1}}{f_{3}}\right)_{\lambda=\lambda_{j}}\right\}= \\
=\left\{\frac{1}{\lambda_{j}^{4}}\left(\frac{f_{4}}{f_{3}}\right)_{\lambda=\lambda_{j}}-\frac{1}{\lambda_{k}^{4}}\left(\frac{f_{4}}{f_{3}}\right)_{\lambda=\lambda_{k}}\right\} .
\end{gathered}
$$

Similarly, for each pair of measured natural frequencies Eq. (7) can be written as,

$$
\frac{1}{K_{x}} \mathbf{T}_{i}^{*}+\frac{1}{K_{y}} \mathbf{U}_{i}^{*}+\frac{1}{K_{z}} \mathbf{V}_{i}^{*}=\mathbf{S}_{i}^{*} ;
$$

where the subscript $i$ represents each combination of $j$ and $k$. Equation (8) is a linear equation in three unknowns $K_{x}, K_{y}$ and $K_{z} ; T_{i}^{*}, U_{i}^{*}, V_{i}^{*}$ and $S_{i}^{*}$ are the coefficients which can be computed from the pair of frequency $\lambda=\lambda_{j}, \lambda_{k}$. The unknown joint stiffness parameters can then be obtained using multi linear regression technique.

\section{SIMULATION: 2-P AND 3-P MODEL}

Equation (6), which is a frequency equation for the 3parameter joint model of the cantilever beam, was considered here to help study the effect of cross coupling parameter on natural frequencies of system. For $K_{z}=\infty$, Eq. (6) reduces to an equation of the 2-parameter model. It can be observed from the equation that the natural frequencies of the system depend on material and geometrical parameters of beam (sub-system T) and joint stiffness parameters. Material and geometrical properties can be estimated accurately. However, joint needs to be modeled accurately in order to estimate correct natural frequencies.

\subsection{Numerical Simulation: Sensitivity Study}

Equation (6) gives natural frequencies of a system if beam and joint parameters are known. In the forward analysis, sample values of joint stiffness parameters were assumed and natural frequencies were estimated, the objective was to study the effect of cross coupling parameter on natural frequencies. Figures $5 \mathrm{a}$ to $5 \mathrm{e}$ shows the variation of first five natural frequencies with cross coupling stiffness $K_{z}$. Further, a ratio $\alpha=K_{z} /\left(K_{x} \cdot K_{y}\right)$ is defined to indicate the relative strength of the cross coupling terms in the joint matrix.

It can be concluded from the sensitivity plot shown in Figs. 5a to 5e and Table 1 that:

- As the cross coupling stiffness tends to zero, the joint rigidity vanishes and frequencies approach towards the ideal frequencies of the free-free beam.

- When cross coupling stiffness tends to go to infinity, the 3-P model tends to 2-P model and for large values of the $K_{x}$ and $K_{y}$ frequencies approaches to ideal values of cantilever beam.

- Natural frequencies are insensitive to $K_{z}$ for $\alpha>1$ and are very close to those estimated from the 2-P model.

- For $\alpha<1$, the estimated natural frequencies deviate considerably from those estimated from the 2-P model.

- A region for the 3-P model can be defined between ideal values of the cantilever beam and $\alpha<1$.

\subsection{Numerical Simulation: Parameter Estimation}

A characteristic equation for the 2-parameter and 3parameter model with unknown non-dimensional stiffness parameters is given in Eqs. (2) and (6), respectively. For the numerical simulation, Eqs. (2) to (8) were used. For sample values of $K_{x}, K_{y}$ in the case of 2-P and $K_{x}, K_{y}$, and $K_{z}$ in case of 3-P, the first five natural frequencies were computed from 


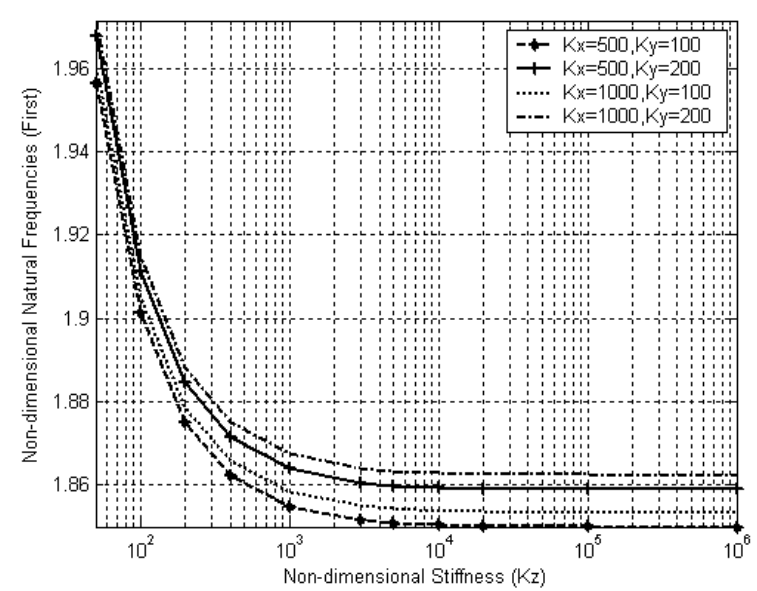

a) The sensitivity of the first natural frequency.

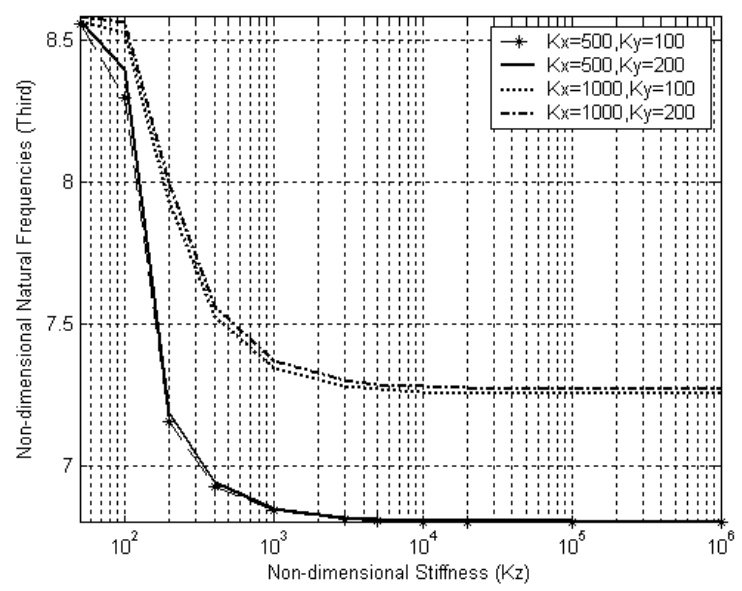

c) The sensitivity of the third natural frequency.

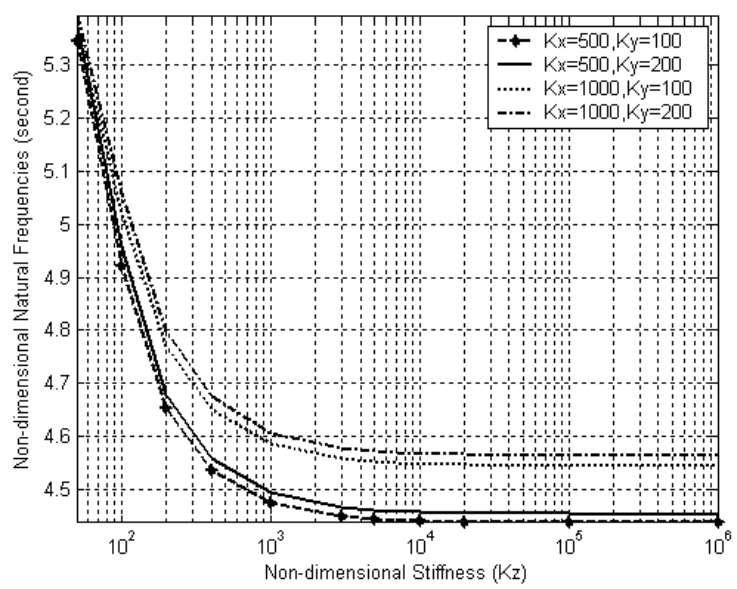

b) The sensitivity of the second natural frequency.

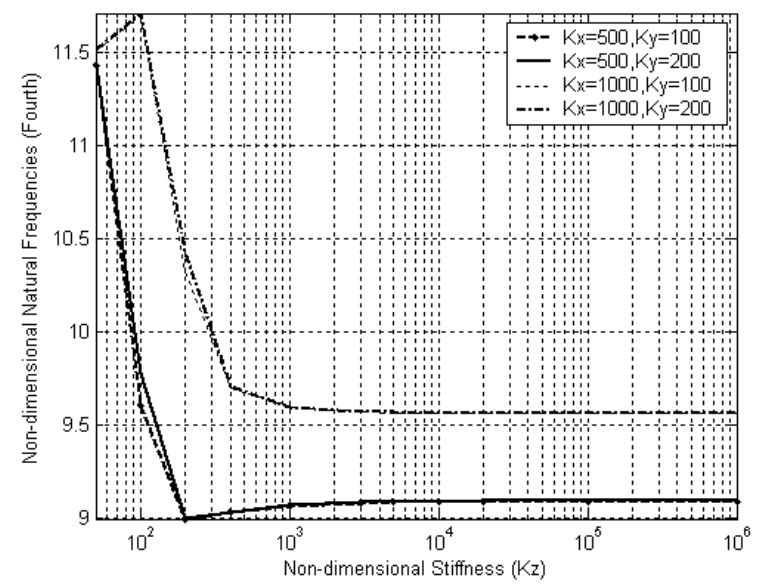

d) The sensitivity of the fourth natural frequency.

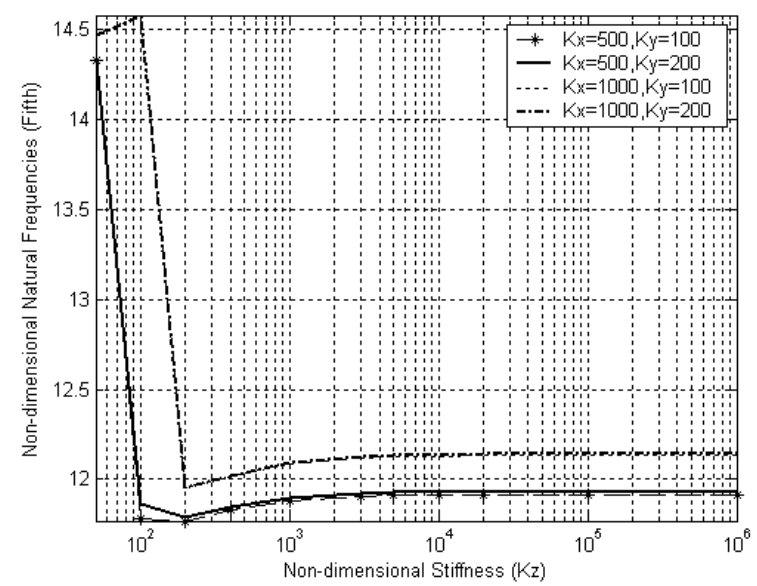

e) The sensitivity of the fifth natural frequency.

Figure 5. The sensitivity of natural frequencies to cross coupling stiffness (Kz).

the FE model. The FE model was discretized into 40 finite elements. The dimensions of the beam were taken as Length $=1 \mathrm{~m}$, cross section $=2 \times 10^{-4} \mathrm{~m}^{2}$; density $=7850 \mathrm{Kg} \mathrm{m}^{-3}$ and Young modulus $\mathrm{E}=207 \mathrm{GPa}$. A computer program was developed in MATLAB $\mathbb{R}{ }^{23}$ for estimating the natural frequencies; details of FE modeling have not been presented for the sake of brevity. The computed frequencies were treated as measured natural frequencies and non-dimensional stiffness parameters were estimated using Eqs. (2) to (8) for the 2-P and 3-P model; the simulation results are given in Table 2 and Table 3. Furthermore, a ratio $\alpha=K_{z} /\left(K_{x} \cdot K_{y}\right)$ was defined for 3-parameter model to indicate the relative strength of the cross coupling terms in the joint matrix.

It can be observed from the simulation results presented in Table 2 that if the system does not have the presence of cross coupling, then both the 2-P and 3-P give good estimates of $K_{x}$ and $K_{y}$. However, estimated $K_{z}$ gives unrealistic values which may confirm non-presence of cross coupling in the system. 
Table 1. Simulation results with sample values of non-dimensional stiffness parameters using the 2-P and 3-P model.

\begin{tabular}{|c|c|c|c|c|c|c|}
\hline \multirow{2}{*}{$\begin{array}{c}\text { Joint } \\
\text { Model }\end{array}$} & \multirow{2}{*}{$\begin{array}{c}\text { Sample values } \\
\text { of stiffness } \\
\text { parameters }\end{array}$} & \multicolumn{5}{|c|}{ Non-dimensional natural frequencies } \\
\hline & & $f_{r 1}$ & $f_{r 2}$ & $f_{r 3}$ & $f_{r 4}$ & $f_{r 5}$ \\
\hline $2-\mathrm{P}$ & $\begin{array}{l}K_{x}=100 \\
K_{y}=100\end{array}$ & 1.8228 & 3.7746 & 5.8178 & 8.6870 & 11.7608 \\
\hline $3-\mathrm{P}$ & $\begin{array}{c}K_{x}=100 \\
K_{y}=100 \\
K_{z}=2000 \\
(\alpha=0.2)\end{array}$ & 1.8252 & 3.7811 & 5.8124 & 8.6816 & 11.7567 \\
\hline $3-\mathrm{P}$ & $\begin{array}{c}K_{x}=100 \\
K_{y}=100 \\
K_{z}=20000 \\
(\alpha=2)\end{array}$ & 1.8230 & 3.7752 & 5.8173 & 8.6864 & 11.7603 \\
\hline $2-\mathrm{P}$ & $\begin{array}{l}K_{x}=500 \\
K_{y}=200\end{array}$ & 1.8589 & 4.4534 & 6.8073 & 9.0964 & 11.9326 \\
\hline $3-\mathrm{P}$ & $\begin{array}{c}K_{x}=500 \\
K_{y}=200 \\
K_{z}=10000 \\
(\alpha=0.1)\end{array}$ & 1.8594 & 4.4573 & 6.8113 & 9.0937 & 11.9289 \\
\hline $3-\mathrm{P}$ & $\begin{array}{c}K_{x}=500 \\
K_{y}=200 \\
K_{z}=500000 \\
(\alpha=5)\end{array}$ & 1.8589 & 4.4535 & 6.8074 & 9.0963 & 11.9326 \\
\hline
\end{tabular}

Table 2. Simulation results with sample values of non-dimensional stiffness parameters (2-P model).

\begin{tabular}{||c|c|c|c||}
\hline $\begin{array}{c}\text { Sample values } \\
\text { of } K_{x} \text { and } K_{y}\end{array}$ & $\begin{array}{c}\text { Non dimensional } \\
\text { natural frequency }\end{array}$ & $\begin{array}{c}\text { Estimated } K_{x} \\
\text { and } K_{y} \text { with 2-P } \\
\text { model }\end{array}$ & $\begin{array}{c}\text { Estimated } K_{x}, \\
K_{y} \text { and } K_{z} \text { with } \\
\text { 3-P model }\end{array}$ \\
\hline$K_{x}=100$ & $f_{r 1}=1.8066$ & $K_{x}=99.95$ & $K_{x}=100$ \\
$K_{y}=50$ & $f_{r 2}=3.7678$ & $K_{y}=50.03$ & $K_{y}=50.07$ \\
& $f_{r 3}=5.8097$ & & $K_{z}=-8.11 \times 10^{5}$ \\
& $f_{r 4}=8.6564$ & & \\
& $f_{r 5}=11.7153$ & & $K_{x}=499.99$ \\
$K_{x}=500$ & $f_{r 1}=1.85$ & $K_{x}=500.154$ & $K_{y}=100.24$ \\
$K_{y}=100$ & $f_{r 2}=4.4368$ & $K_{y}=100.27$ & $K_{z}=-5.67 \times 10^{6}$ \\
& $f_{r 3}=6.8022$ & & \\
& $f_{r 4}=9.0929$ & & $K_{x}=1000.82$ \\
& $f_{r 5}=11.9156$ & & $K_{y}=510.06$ \\
$K_{x}=1000$ & $f_{r 1}=1.8679$ & $K_{x}=1018.644$ & \\
$K_{y}=500$ & $f_{r 2}=4.5755$ & $K_{y}=517.31$ & \\
& $f_{r 3}=7.2837$ & & \\
& $f_{r 4}=9.5658$ & & \\
\hline
\end{tabular}

Table 3 shows the simulation results using the 3-parameter model; it can be observed that with an increase in crosscoupling stiffness, $\alpha$ increases and the natural frequencies of the 3-parameter model shifts towards the values of the 2parameter model, which means that the 3-parameter model with its large $\alpha$ is almost the same as the 2-parameter model. Stiffness parameters are estimated here from the so called measured frequency data. Table 3 shows the estimates for different cross coupling ratios. It can be seen that estimates for direct stiffness parameters are very good using both models when $\alpha \geq 1$ but that for the cross coupling term is good only for $\alpha<<1$. The presence of cross coupling effect in a joint system can be parametrically estimated from 3-P model, provided relative strength of cross coupling i.e., $\alpha$ is less than 1 , in such case the 2-P model gives incorrect estimates of $K_{x}$ and $K_{y}$.

The variation of translational stiffness $K_{x}$ and $K_{y}$ with relative strength of cross coupling $\alpha=\left(K_{z} / K_{x} \cdot K_{y}\right)$ as shown in Figs. 6 to 8 shows that for $\alpha \geq 1$, estimated $K_{x}$ and $K_{y}$ values approaches towards exact values, however $K_{z}$ gives unrealistic values.

One can thus conclude that a 3-parameter model can achieve

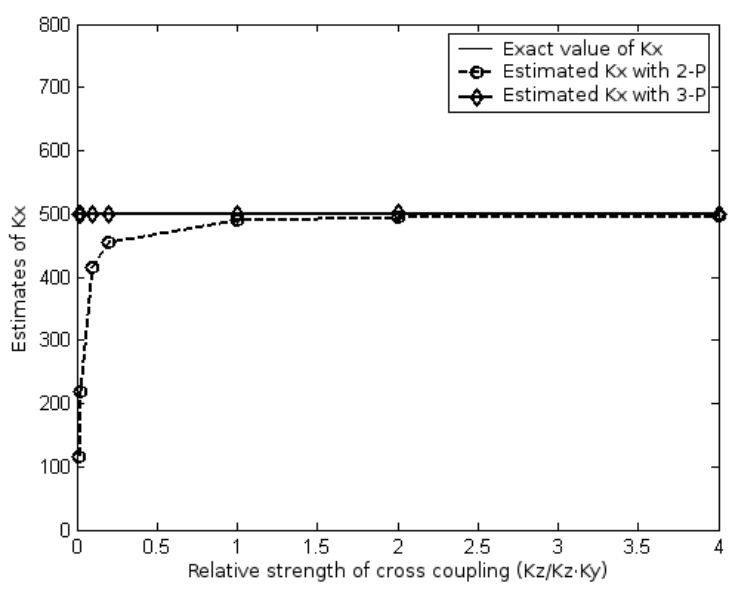

Figure 6. The variation of $K_{x}$ with the relative strength of cross coupling $\alpha$.

a good estimate only when the cross coupling stiffness is small or for $\alpha<<1$. For $\alpha$ close to or higher than 1, it is better to adopt the 2-parameter model as opposed to the 3-parameter model. 
Table 3. Simulation results with sample values of non-dimensional stiffness parameters (3-P model).

\begin{tabular}{|c|c|c|c|}
\hline $\begin{array}{c}\text { Sample values } \\
\text { of } K_{x}, K_{y} \text {, and } K_{z}\end{array}$ & $\begin{array}{l}\text { Non dimensional } \\
\text { natural frequency }\end{array}$ & $\begin{array}{c}\text { Estimated } K_{x} \\
\text { and } K_{y} \text { with 2-P } \\
\text { model }\end{array}$ & $\begin{array}{c}\text { Estimated } K_{x}, \\
K_{y} \text { and } K_{z} \text { with } \\
\text { 3-P model }\end{array}$ \\
\hline $\begin{array}{c}K_{x}=1000 \\
K_{y}=500 \\
K_{z}=10000 \\
(\alpha=0.02)\end{array}$ & $\begin{array}{l}f_{r 1}=1.8684 \\
f_{r 2}=4.5798 \\
f_{r 3}=7.2930 \\
f_{r 4}=9.5680 \\
f_{r 5}=12.141\end{array}$ & $\begin{array}{l}K_{x}=408.75 \\
K_{y}=223.47\end{array}$ & $\begin{array}{c}K_{x}=999.85 \\
K_{y}=506.28 \\
K_{z}=10038.73\end{array}$ \\
\hline $\begin{array}{c}K_{x}=500 \\
K_{y}=100 \\
K_{z}=5000 \\
(\alpha=0.1)\end{array}$ & $\begin{array}{c}f_{r 1}=1.8510 \\
f_{r 2}=4.4443 \\
f_{r 3}=6.8100 \\
f_{r 4}=9.0876 \\
f_{r 5}=11.9082\end{array}$ & $\begin{array}{l}K_{x}=414.58 \\
K_{y}=84.941\end{array}$ & $\begin{array}{l}K_{x}=500.034 \\
K_{y}=100.363 \\
K_{z}=5021.04\end{array}$ \\
\hline $\begin{array}{c}K_{x}=500 \\
K_{y}=100 \\
K_{z}=15000 \\
(\alpha=0.3)\end{array}$ & $\begin{array}{l}f_{r 1}=1.8503 \\
f_{r 2}=4.4393 \\
f_{r 3}=6.8048 \\
f_{r 4}=9.0911 \\
f_{r 5}=11.9131\end{array}$ & $\begin{array}{c}K_{x}=471.79 \\
K_{y}=95.38\end{array}$ & $\begin{array}{l}K_{x}=499.88 \\
K_{y}=99.991 \\
K_{z}=14233.6\end{array}$ \\
\hline $\begin{array}{c}K_{x}=500 \\
K_{y}=100 \\
K_{z}=50000 \\
(\alpha=1)\end{array}$ & $\begin{array}{c}f_{r 1}=1.8501 \\
f_{r 2}=4.4375 \\
f_{r 3}=6.8030 \\
f_{r 4}=9.0923 \\
f_{r 5}=11.9148\end{array}$ & $\begin{array}{c}K_{x}=490.96 \\
K_{y}=98.74\end{array}$ & $\begin{array}{c}K_{x}=500.28 \\
K_{y}=100.36 \\
K_{z}=60648.39\end{array}$ \\
\hline $\begin{array}{c}K_{x}=1000 \\
K_{y}=500 \\
K_{z}=500000 \\
(\alpha=1)\end{array}$ & $\begin{array}{c}f_{r 1}=1.8679 \\
f_{r 2}=4.5756 \\
f_{r 3}=7.2839 \\
f_{r 4}=9.5659 \\
f_{r 5}=12.1463\end{array}$ & $\begin{array}{l}K_{x}=987.51 \\
K_{y}=500.61\end{array}$ & $\begin{array}{c}K_{x}=1000.28 \\
K_{y}=506.96 \\
K_{z}=3.423 \times 10^{6}\end{array}$ \\
\hline $\begin{array}{c}K_{x}=500 \\
K_{y}=100 \\
K_{z}=100000 \\
(\alpha=2)\end{array}$ & $\begin{array}{c}f_{r 1}=1.8501 \\
f_{r 2}=4.4371 \\
f_{r 3}=6.8026 \\
f_{r 4}=9.0926 \\
f_{r 5}=11.9152\end{array}$ & $\begin{aligned} K_{x} & =495.11 \\
K_{y} & =99.71\end{aligned}$ & $\begin{array}{c}K_{x}=500.61 \\
K_{y}=100.86 \\
K_{z}=-2.56 \times 10^{5}\end{array}$ \\
\hline $\begin{array}{c}K_{x}=1000 \\
K_{y}=500 \\
K_{z}=1000000 \\
(\alpha=2)\end{array}$ & $\begin{array}{c}f_{r 1}=1.8679 \\
f_{r 2}=4.5755 \\
f_{r 3}=7.2838 \\
f_{r 4}=9.5658 \\
f_{r 5}=12.1463\end{array}$ & $\begin{array}{l}K_{x}=1007.61 \\
K_{y}=511.485\end{array}$ & $\begin{array}{c}K_{x}=1001.51 \\
K_{y}=511.171 \\
K_{z}=-2.25 \times 10^{5}\end{array}$ \\
\hline
\end{tabular}

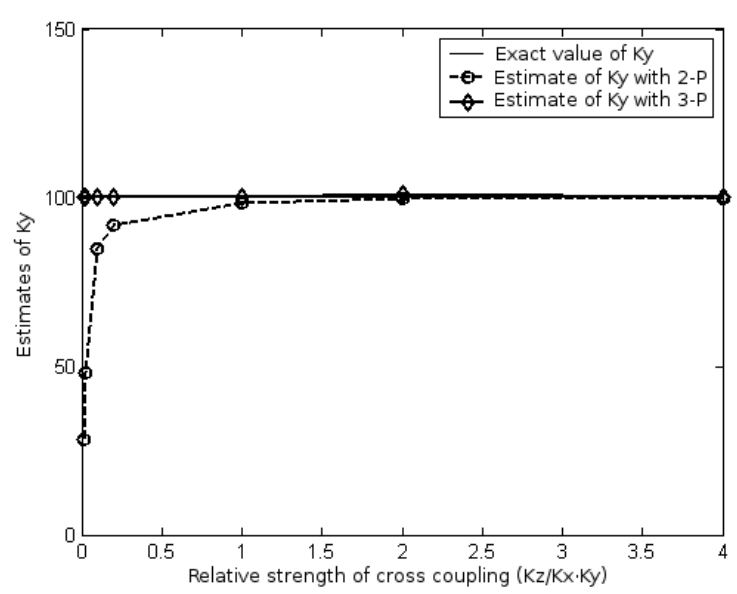

Figure 7. The variation of $K_{y}$ with the relative strength of cross coupling $\alpha$.

Furthermore, the accuracy of joint stiffness estimation using the 2-parameter model is demonstrated using the data presented by W. L. Li, ${ }^{5}$ wherein the unknown joint stiffness parameters were identified using a so-called reduced order characteristic polynomial (ROCP). The joint stiffness was identified using different combinations of the measured natural frequencies and averages of the identified results have been taken. The results obtained using the method proposed by W. L. $\mathrm{Li}^{5}$

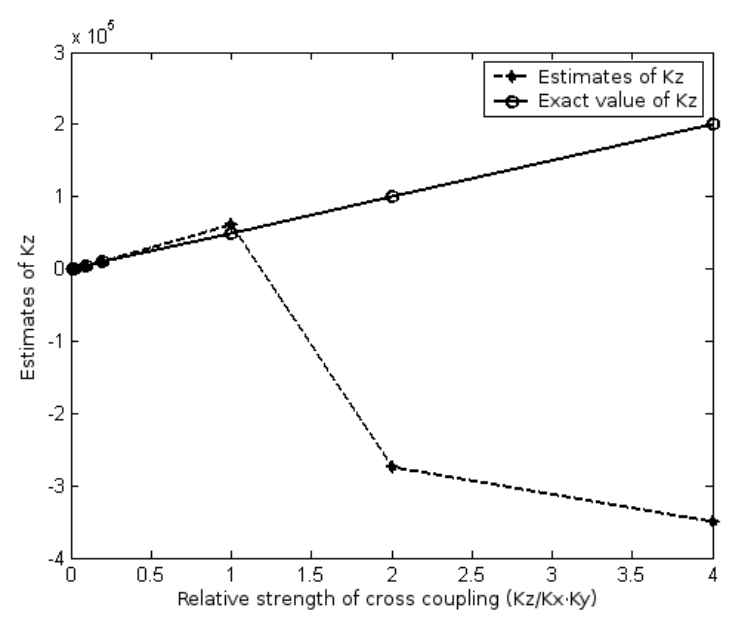

Figure 8. The variation of $K_{z}$ with the relative strength of cross coupling $\alpha$.

are compared with the results that were obtained using the method demonstrated in the present work. Table 4 shows the comparison and it can be observed that the estimated results using the sub-structure synthesis (SSS) method are very much accurate than those obtained using ROCP.

It can be seen that at least three natural frequencies are needed to estimate joint stiffness parameters using the 2-P model. In case of the 3-P model, a minimum of four natu- 


Table 4. A comparison of estimated joint stiffness parameters using ROCP and sub-structure synthesis (SSS).
\begin{tabular}{|c|c|c|c|c|c|}
\hline $\begin{array}{c}\text { Exact values of } \\
\text { stiffness } \\
\text { parameters }\end{array}$ & $\begin{array}{c}\text { Computed } \\
\text { Natural } \\
\text { Frequencies } \\
\text { (Hz) }\end{array}$ & $\begin{array}{c}\text { Estimated } \\
\text { stiffness } \\
\text { parameters } \\
\text { using ROCP }\end{array}$ & $\begin{array}{c}\text { Percentage } \\
\text { error in } \\
\text { estimation }\end{array}$ & $\begin{array}{c}\text { Estimated } \\
\text { stiffness } \\
\text { parameters } \\
\text { using SSS }\end{array}$ & $\begin{array}{c}\text { Percentage } \\
\text { error in } \\
\text { estimation }\end{array}$ \\
\hline$K_{t}=10^{5}$ & $\begin{array}{c}f_{r 1}=9.7900 \\
f_{r 2}=50.150\end{array}$ & $K_{t}=99947$ & 0.053 & $K_{t}=99964$ & 0.036 \\
$K_{r 3}=114.84$ & & & & \\
$K_{r 4}=236.41$ & $K_{r}=9333$ & 6.67 & $K_{r}=10001.7$ & 0.017 \\
\hline$f_{r 5}=427.00$ & & & & \\
\hline
\end{tabular}

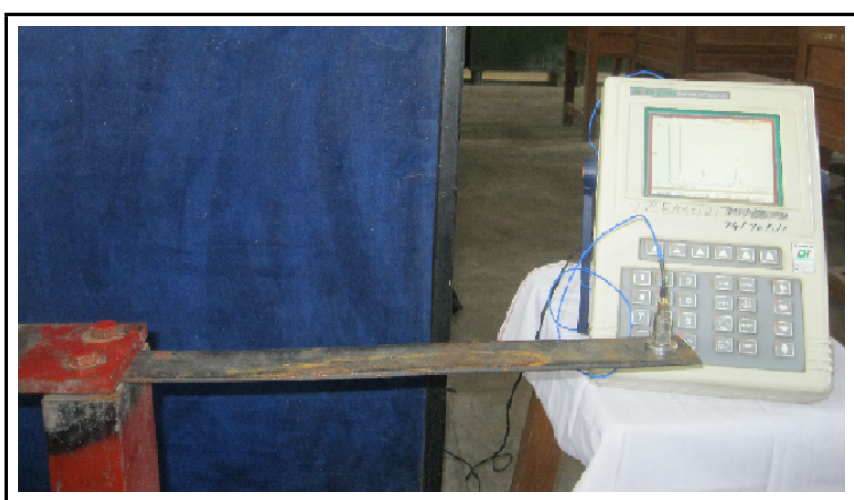

a)

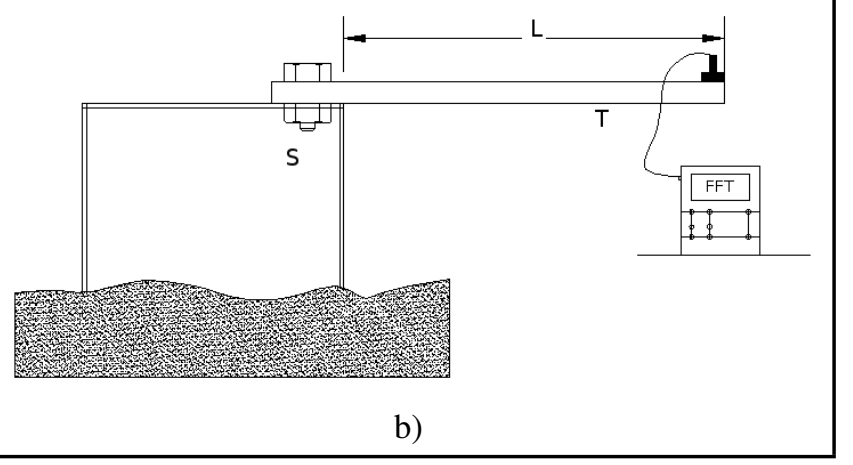

Figure 9. (a) The experimental setup (b) The schematic diagram of the beam with a bolted joint.

ral frequencies are required. In order to determine the optimum number of natural frequencies required for the best estimates of stiffness parameters, a convergence study was carried out wherein more than the minimum required natural frequencies were used. For sample values of the stiffness parameter, the first seven natural frequencies were computed from the FE model. These frequencies were then used in different sets to estimate joint parameters by using the 2-P model. Table 5 shows the estimated stiffness parameters using different sets of natural frequencies.

A similar study was carried out using 3-P model; the results are presented in Table 6 . It can be observed from Table 5 and Table 6 that the estimated stiffness parameters using all sets of the natural frequencies are very close to the exact values.

Since higher modes are generally difficult to measure, it is therefore suggested to use the minimum required number of lower modes for estimating of joint stiffness parameters.

\section{EXPERIMENTAL CASE STUDY}

A test set up for measuring the vibration response of a cantilever beam clamped with a bolt to a structure is shown in Fig. 9.
The beam was excited using an impulse and the free vibration response was measured using B \& K 4370 (sensitivity $100 \mathrm{pC} / \mathrm{g}$ ) accelerometer and FFT analyzer (DI-22, Diagnostic Instruments, UK). A data block size of 2048 samples with 800 spectral lines was used to measure the natural frequencies of beam. The geometrical and material properties of the beam are listed in Table 7.

The values of the material parameters were obtained by testing a beam under free-free condition and updating the corresponding FE model for measured natural frequencies. Two dimensional beam elements were used in the FE modeling and a program was developed in MATLAB $R$ to compute the natural frequencies of the free-free beam; the density of the beam was obtained by measuring its mass and the modulus of elasticity was obtained by updating its value in FE model to validate the measured natural frequencies. Table 8 shows the measured and computed (FEM) natural frequencies of the beam with updated material properties under the unconstrained (free-free) condition.

The vibration response of a cantilever beam clamped with bolts was measured using an FFT analyzer. In order to improve the accuracy in measurement (by reducing resolution error), different frequency bands were selected for the FFT analyzer e.g., for the measurement of the first two natural frequencies, a frequency band of 0-200 Hz was selected is shown in Fig. 10. For higher frequencies a frequency band of $0-1000 \mathrm{~Hz}$ was selected as shown in Fig. 11. The measured natural frequencies at the resonance peaks of frequency spectrum are given in Table 9.

The vibration response of a cantilever beam clamped with bolts was measured using an FFT analyzer. In order to improve the accuracy in measurement (by reducing resolution error), different frequency bands were selected for the FFT analyzer e.g., for the measurement of the first two natural frequencies, a frequency band of 0-200 Hz was selected as shown in Fig. (10). For higher frequencies a frequency band of $0-1000 \mathrm{~Hz}$ was selected as shown in Fig.(11).

\subsection{A Two Parameter Model}

The linear parameters of the joint, i.e., translational stiffness $K_{t}$ and rotational stiffness $K_{r}$ are identified using first four natural frequencies only, which are given in Table 10. The support parameters are the most important in the lower modes and are less significant in higher modes, for this reason first four modes were chosen in the identification procedure. ${ }^{15}$ The linear equation with two unknowns $K_{x}$ and $K_{y}$ was obtained from Eq. (3) by using the first four measured natural frequencies. Equation (5) was then used for the best estimate of 
Table 5. Estimated stiffness parameters using different sets of natural frequencies (2-P model).

\begin{tabular}{||c|c|c|c|c||}
\hline \multirow{2}{*}{$\begin{array}{c}\text { Sample Values } \\
\text { of } K_{x} \text { and } K_{y}\end{array}$} & $\begin{array}{c}\text { Computed } \\
\text { Natural }\end{array}$ & $\begin{array}{c}\text { Set of natural frequencies } \\
\text { for joint stiffness estimation }\end{array}$ & \multicolumn{2}{|c|}{$\begin{array}{c}\text { Estimated Joint Stiffness } \\
\text { Parameters }\end{array}$} \\
\cline { 4 - 5 } & Frequencies & & $K_{x}$ & $K_{y}$ \\
\hline$K_{x}=500$ & $f_{r 1}=1.8500$ & $f_{r 1} ; f_{r 2} ; f_{r 3}$ & 500.741 & 100.39 \\
& $f_{r 2}=4.4368$ & $f_{r 1} ; f_{r 2} ; f_{r 3} ; f_{r 4}$ & 500.612 & 100.37 \\
$K_{y}=100$ & $f_{r 3}=6.8022$ & $f_{r 1} ; f_{r 2} ; f_{r 3} ; f_{r 4} ; f_{r 5}$ & 500.074 & 100.25 \\
& $f_{r 4}=9.0929$ & $f_{r 1} ; f_{r 2} ; f_{r 3} ; f_{r 4} ; f_{r 5} ; f_{r 6}$ & 499.750 & 100.16 \\
& $f_{r 5}=11.9156$ & $f_{r 1} ; f_{r 2} ; f_{r 3} ; f_{r 4} ; f_{r 5} ; f_{r 6} ; f_{r 7}$ & 499.513 & 100.07 \\
& $f_{r 6}=14.9452$ & & & \\
\hline
\end{tabular}

Table 6. Estimated stiffness parameters using different sets of natural frequencies (3-P model).

\begin{tabular}{|c|c|c|c|c|c||}
\hline \hline Sample & Computed & Set of natural & \multicolumn{3}{|c|}{ Estimated Joint Stiffness Parameters } \\
\cline { 5 - 6 } Values of $K_{x}$ & Natural & frequencies for joint & $K_{x}$ & $K_{y}$ & $K_{z}$ \\
and $K_{y}$ & Frequencies & stiffness estimation & & & \\
\hline$K_{x}=500$ & $f_{r 1}=1.8510$ & $f_{r 1} ; f_{r 2} ; f_{r 3} ; f_{r 4}$ & 500.00 & 100.35 & 5013.01 \\
& $f_{r 2}=4.4443$ & & & & \\
$K_{y}=100$ & $f_{r 3}=6.8100$ & $f_{r 1} ; f_{r 2} ; f_{r 3} ; f_{r 4} ; f_{r 5}$ & 500.07 & 100.37 & 5030.87 \\
& $f_{r 4}=9.0876$ & $f_{r 1} ; f_{r 2} ; f_{r 3} ; f_{r 4} ; f_{r 5} ; f_{r 6}$ & 500.07 & 100.37 & 5032.41 \\
$K_{z}=5000$ & $f_{r 5}=11.9082$ & & & & \\
& $f_{r 6}=14.9389$ & $f_{r 1} ; f_{r 2} ; f_{r 3} ; f_{r 4} ; f_{r 5} ;$ & 500.07 & 100.37 & 5033.53 \\
& $f_{r 7}=18.0283$ & $f_{r 6} ; f_{r 7}$ & & & \\
\hline
\end{tabular}

Table 7. The dimensions and material properties of the beam.

\begin{tabular}{|c|c|}
\hline Dimensions & Material Properties \\
\hline $\begin{array}{c}\text { Cross sectional area: } \\
(0.05 \times 0.005) \mathrm{m}^{2}\end{array}$ & Elastic modulus, $E: 207 \mathrm{GPa}$ \\
\hline Length of beam: $0.423 \mathrm{~m}$ & Density, $\rho: 7850 \mathrm{Kg} \mathrm{m}^{-3}$ \\
\hline
\end{tabular}

Table 8. Comparison of the natural frequency of the beam under free-free condition.

\begin{tabular}{|c|c|c|c|c||}
\hline Natural frequencies (Hz) & $f_{r 1}$ & $f_{r 2}$ & $f_{r 3}$ & $f_{r 4}$ \\
\hline Measured & 105 & 290.25 & 571.25 & 944 \\
FEM & 105.27 & 290.71 & 570.85 & 943.06 \\
\hline
\end{tabular}

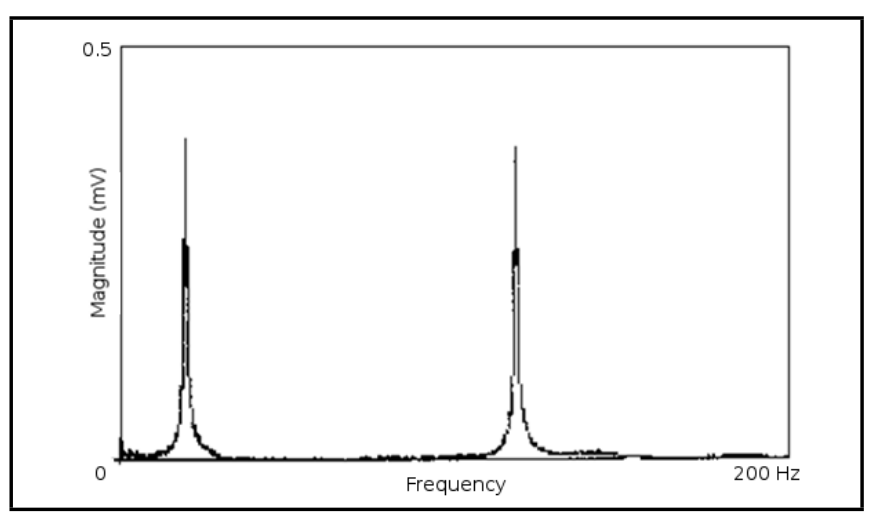

Figure 10. Vibration response of the cantilever beam using a real time FFT analyzer at $0-200 \mathrm{~Hz}\left(1 \mathrm{mV}=10^{-2} \mathrm{~g}\right)$.

non-dimensional stiffness parameters using non dimensional frequencies. The estimated non dimensional stiffness parameters and corresponding linear joint parameters are given in Table 10.

Next, the identified joint parameters were incorporated into the FE model and the natural frequencies were computed. These frequencies were then compared with the experimentally measured natural frequencies. Table 11 show the comparison and it can be observed that there was considerable error in the measured and computed natural frequencies. The reasons for error in natural frequencies may be due to insufficient finite elements in FE modeling and over simplification of joint model (Model 1, 2-P model), where cross coupling terms are

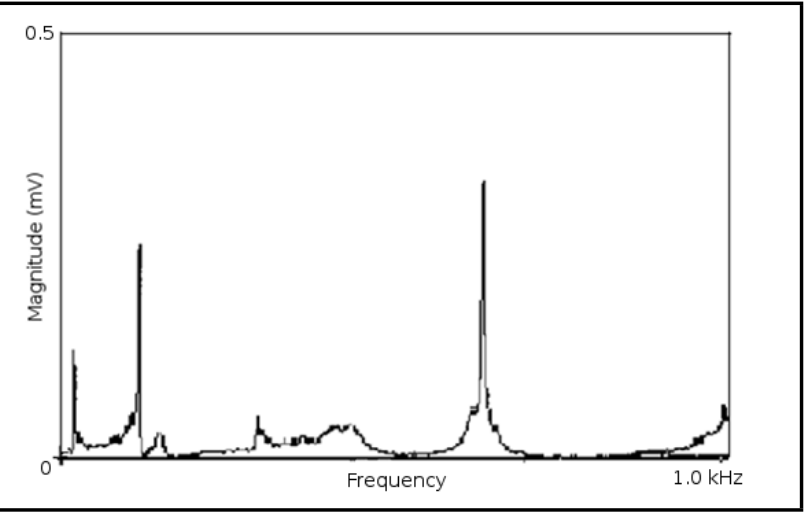

Figure 11. The vibration response of the cantilever beam using a real time FFT analyzer at $0-1 \mathrm{KHz}\left(1 \mathrm{mV}=10^{-2} \mathrm{~g}\right)$.

neglected. The former reason could be excluded easily by increasing number of finite elements; the later reason was studied by using joint Model 2 (the 3-P model).

\subsection{A Three Parameter Model}

The measured natural frequencies given in Table 9 were incorporated into Eqs. (6) to (8) in order to estimate the three parameters of the joint. The identified joint parameters are given in Table 12. Next, the identified joint parameters were incorporated into the FE model and the natural frequencies were computed. These frequencies were then compared with the experimentally measured natural frequencies (Table 13 shows the comparison).

The relative strength of cross coupling term $\alpha$ is,

$$
\alpha=K_{z} /\left(K_{x} \cdot K_{y}\right)=0.0921 \text {. }
$$

The FE model update with the estimated joint parameters gives a better estimate of the natural frequencies than the two parameter model. The ratio $\alpha$ defined in the previous section has the value $<<1$ for the joint demonstrated in this paper. It is evident from simulation and experimentation results that since $\alpha<<1$, the 3-P model gives better estimates of joint parameters than the 2-P model. Although only the first four measured 
Table 9. Measured natural frequencies of a cantilever beam clamped with a bolt.

\begin{tabular}{|c|c|c|c|c|c|}
\hline Mode & $f_{r 1}$ & $f_{r 2}$ & $f_{r 3}$ & $f_{r 4}$ & $f_{r 5}$ \\
\hline Natural frequency (Hz) & 17 & 118 & 335.625 & 628.75 & 994.5 \\
Non-dimensional natural frequency & 1.6068 & 4.2335 & 7.1398 & 9.7724 & 12.2904 \\
\hline
\end{tabular}

Table 10. The identified joint parameters (2-P).

\begin{tabular}{|c|c|}
\hline $\begin{array}{c}\text { Non-dimensional joint } \\
\text { stiffness parameters }\end{array}$ & Joint stiffness parameters \\
\hline$K_{x}=1294.906$ & $K_{t}=1.844 \times 10^{6}\left(\mathrm{~N} \cdot \mathrm{m}^{-1}\right)$ \\
$K_{y}=3.5021$ & $K_{r}=892.601\left(\mathrm{~N} \cdot \mathrm{m} \cdot \mathrm{rad}^{-1}\right)$ \\
\hline
\end{tabular}

Table 11. A comparison of natural frequencies measured experimentally and computed with an updated FE model (2-P).

\begin{tabular}{|c|c|c|c|c|c||}
\hline Mode & $f_{r 1}$ & $f_{r 2}$ & $f_{r 3}$ & $f_{r 4}$ & $f_{r 5}$ \\
\hline Measured natural frequencies (Hz) & 17 & 118 & 335.625 & 628.75 & 994.5 \\
Natural frequencies with updated FE model (Hz) & 15.745 & 113.92 & 327.7 & 615.91 & 982.91 \\
Percentage Error (\%) & 7.38 & 3.46 & 2.36 & 2.04 & 1.16 \\
\hline
\end{tabular}

Table 12. The identified joint parameters (3-P).

\begin{tabular}{|c|c|}
\hline $\begin{array}{c}\text { Non-dimensional joint } \\
\text { stiffness parameters }\end{array}$ & Joint stiffness parameters \\
\hline$K_{x}=1492.129$ & $K_{t}=2.12 \times 10^{6}\left(\mathrm{~N} \cdot \mathrm{m}^{-1}\right)$ \\
$K_{y}=4.679$ & $K_{r}=1192.564\left(\mathrm{Nm} \cdot \mathrm{rad}^{-1}\right)$ \\
$K_{z}=643.633$ & $K_{r t}=3.88 \times 10^{5}\left(\mathrm{~N} \cdot \mathrm{rad}^{-1}\right)$ \\
\hline
\end{tabular}

Table 13. A comparison of the natural frequency measured experimentally and computed with an updated FE model (3-P).

\begin{tabular}{|c|c|c|c|c|c||}
\hline Mode & $f_{r 1}$ & $f_{r 2}$ & $f_{r 3}$ & $f_{r 4}$ & $f_{r 5}$ \\
\hline Measured natural frequencies (Hz) & 17 & 118 & 335.625 & 628.75 & 994.5 \\
Natural frequencies computed with updated FE model (Hz) & 16.96 & 117.56 & 337.7 & 652.77 & 1037.3 \\
\% Error & 0.23 & 0.37 & -0.618 & -3.82 & -4.3 \\
\hline
\end{tabular}

Table 14. Estimates of the joint stiffness parameters and estimation errors with a $\pm 0.1 \%$ frequency perturbation.

\begin{tabular}{||c|c|c|c||}
\hline Joint Parameters & $K_{x}$ & $K_{y}$ & $K_{z}$ \\
\hline Identified Values & 1492.129 & 4.679 & 643.633 \\
$\begin{array}{c}\text { Estimated with }+0.1 \% \\
\text { frequency perturbation }\end{array}$ & 1454.308 & 4.705 & 625.525 \\
$\begin{array}{c}\text { Estimated with }-0.1 \% \\
\text { frequency perturbation } \\
\text { Average \% error }\end{array}$ & 1532.485 & 4.6544 & 662.992 \\
\hline
\end{tabular}

natural frequencies are considered in regression analysis, the fifth measured natural frequency is also close to the one computed with FE model. This shows that the higher natural frequencies that were not considered in regression analysis also validate the proposed algorithm.

For practical measurement of natural frequencies, the effect of the measurement noise also needs to be considered. The major source of error with FFT analyzers is spectral resolution error, which depends on data block size for FFT processing. For a typical 2048 data block size, 800 spectral lines are displayed in the given frequency range $f$, which means two adjacent frequency data were separated by $f / 800$. This is known as resolution error, as any frequency falling within two adjacent frequency lines cannot be displayed at the actual position but rather at one of these two frequency positions. The error in the measurement then becomes $f /(2 * 800)=0.0625 \%$. Over and above this resolution error, there may also be random noise. Hence, the measured frequency values given in Table 9 are perturbed by $\pm 0.1 \%$ to test the effect of measurement error on the estimation procedure. Table 14 shows estimates of joint stiffness parameters with perturbed natural frequencies. The average error in identified joint parameters is within 3\%, which shows that the method is robust against measurement error.

\section{CONCLUSIONS}

A new procedure for joint stiffness identification has been proposed in this work. The procedure is based on the natural frequency measurement and hence is very much convenient in practical applications. Using the method of sub-structure synthesis, a frequency equation in terms of the joint stiffness parameters is developed for both two parameter and three parameter structural joint. With the measured natural frequencies, one can obtain an over determined set of equations, which is then processed through multi-linear regression to obtain the best estimates of the joint parameters. It is shown that the procedure gives accurate estimates for a wide range of stiffness values. Unknown joint stiffness parameters are identified for physical structure using both 2-parameter and 3-parameter model. It was observed for the demonstrated structure that the 3-parameter model gives better estimate than 2-parameter model. However, the selection of appropriate model of joint can be suggested by demonstrated procedure. The natural frequencies estimated with the updated FE model are very close to experimentally measured natural frequencies, moreover the frequencies not considered in regression analysis also validate the model and hence proposed method can be extended for modeling the joints using FE method. Although the demon- 
stration is for a cantilever beam with end supports, the procedure can be used for stiffness identification of various other joints such as lap joint etc.

\section{REFERENCES}

1 Wang, J. H. and Sas, P. A method for identifying parameters of mechanical joints, Journal of Applied Mechanics, 57, 337-342, (1990). http://dx.doi.org/10.1115/1.2891994

2 Ren, Y. and Beards, C. F. Identification of 'effective' linear joints using coupling and joint identification techniques, Journal of Vibration and Acoustics, 120, 331-338, (1998). http://dx.doi.org/10.1115/1.2893835

3 Nobari, A. S., Robb, D. A., and Ewins, D. J. A new modal-based method for structural dynamic model updating and joint identification, Proceedings of the 10th International Modal Analysis Conference, San Diego, 1, 741-750, (1992).

4 Ma, X., Bergman, L., and Vakakis, A. Identification of bolted joints through laser vibrometry, Journal of Sound and Vibrations, 246 (3), 441-460, (2001). http://dx.doi.org/10.1006/jsvi.2001.3573

5 Li, W. L. A New Method for Structural Model Updating and Joint Stiffness Identification, Mechanical Systems and Signal Processing, 16 (1), 155-167, (2002). http://dx.doi.org/10.1006/mssp.2000.1339

6 Mottershed, J. E., Friswell, M. I., Ng, G. H. T., and Brandon, J. A. Geometric parameters for finite element model updating of joints and constraints, Mechanical Systems and Signal Processing, 10 (2), 171-182, (1996). http://dx.doi.org/10.1006/mssp.1996.0012

7 Yang, K. T. and Park, Y. S. Joint structural parameter identification using a subset of frequency response function measurement, Mechanical Systems and Signal Processing, 7, 509-530, (1993). http://dx.doi.org/10.1006/mssp.1993.1030

8 Chen, J. C. and Garba, J. A. Analytical model improvement using modal test results, Am. Inst. Aeronaut. Astronaut Journal, 18, 684-690, (1984). http://dx.doi.org/10.2514/3.50805

9 Minas, C. and Inman, D. J. Matching finite element models to modal data, Journal of Vibrations and Acoustics, 112, 84-92, (1990). http://dx.doi.org/10.1115/1.2930103

10 Mottershead, J. E. and Weixun, S. Correction of joint stiffnesses and constraints for finite element models in structural dynamics, Journal of Applied Mechanics, 60, 117122, (1993). http://dx.doi.org/10.1115/1.2900733

11 Huckelbridge, A. A. and Lawrence, C. Identification of structural interface characteristics using component mode synthesis, Journal of Vibrations, Acoustics Stress and Reliability in Design, 111, 140-147, (1989). http://dx.doi.org/10.1115/1.3269834
12 Piranda, J., Lallement, G., and Cogan, S. Parametric correction of finite element models by minimization of an output residual: improvement of sensitivity method, Proceedings of 9th International Modal Analysis Conference, USA, (1991).

13 Yoshimura, M. Computer-aided design improvement of machine tool structure incorporating joint dynamics data, Ann. CIRP, 28, 241-246, (1979).

14 Pabst, U. and Hagedorn, P. Identification of boundary conditions as a part of model correction, Journal of Sound and Vibration, 182 (4), 565-575, (1995). http://dx.doi.org/10.1006/jsvi.1995.0217

15 Ahmadian, H., Mottershed, J. E., and Friswell, M. I. Boundary condition Identification by Solving Characteristic Equations, Journal of Sound and Vibration, 247 (5), 755-763, (2001). http://dx.doi.org/10.1006/jsvi.2001.3708

16 Yang, K-T and Park, Y-S Joint structural parameter identification using a subset of frequency response function measurements, Mechanical Systems and Signal Processing, 7 (6), 509-530, (1993). http://dx.doi.org/10.1006/mssp.1993.1030

17 Celic, D. and Boltezar, M. Identification of the dynaimc properties of joints using frequency response functions, Journal of Sound and Vibration, 317, 158-174, (2008). http://dx.doi.org/10.1016/j.jsv.2008.03.009

18 Ren, Y. and Beards, C. F. Identification of Joint Properties of a Sub-structure using FRF Data, Journal of Sound and Vibration, 186 (4), 567-587, (1995). http://dx.doi.org/10.1006/jsvi.1995.0469

19 Ren, Y. and Beards, C. F. On the nature of FRF joint identification technique, Proceedings of the 11th International Modal Analysis Conference, Florida, USA, 473-478, (1993).

20 Wang, M., Wang, D., and Zheng, G. Joint dynamic properties identification with partially measured frequency response function, Mechanical Systems and Signal Processing, 27, 499-512, (2012). http://dx.doi.org/10.1016/j.ymssp.2011.09.024

21 Ingole, S. B. and Chatterjee, A. Joint parameter identification of a cantilever beam using sub-structure synthesis and multi-linear regression, Structural Engineering and Mechanics, 45 (4), 423-437, (2013). http://dx.doi.org/10.12989/sem.2013.45.4.423

22 Draper, N. R. and Smith, H. Applied Regression Analysis, John Willey and Sons, Singapore, (1998). http://dx.doi.org/10.1002/9781118625590

23 Gilat, A. MATLAB $\mathrm{R}$ An introduction with applications, John Wiley \& Sons (Asia) Pte.Ltd., Singapore, (2003). 